\title{
LE DÉGOÛT DE L'ABSURDE : PHÉNOMÉNOLOGIE DE L'EXISTENCE DANS LA NAUSÉE DE JEAN-PAUL SARTRE
}

Toute enquête sur le vécu du dégoût n'est jamais sans considérer ou citer - même simplement en passant - La nausée de Jean-Paul Sartre ${ }^{1}$. Ce célèbre roman, qui a été défini comme " phénoménologique » (Contat-Rybalka 1995, 1664 ; De Coorebyter 2005, 14), recèle en effet un remarquable potentiel philosophique, dont le cœur concerne la portée du phénomène du dégoût, dans sa stricte corrélation avec l'existence. Dans ce cadre, le présent travail se propose d'analyser le dégoût dont la nausée sartrienne est expression, en cernant sa signification ontologique, afin de faire émerger le rôle fondamental et fondateur de cette dernière au sein de la pensée de Sartre.

À partir des premières lignes de La nausée, le propos phénoménologique du roman est déclaré. Le personnage principal, Antoine Roquentin, exprime l'exigence de « tenir un journal pour $y$ voir clair » (Sartre 1938, 5, je souligne), soit pour " déterminer exactement l'étendue et la nature » (ibid.) du changement qui a touché la façon dont il perçoit les objets et les personnes qui peuplent son monde. L'attention au thèmes - typiquement phénoménologiques - de la vision et de la conversion du regard (cf. Husserl 1913, §§ 3, 31-32 ; Heidegger 1927, § 7) ne sera dorénavant jamais abandonnée, spécialement pour ce qui concerne la stricte corrélation que ces postures entretiennent avec le vécu du dégoût, laquelle est aussi posée clairement dès l'incipit. En effet, au sein de l'interrogation introspective sur laquelle s'ouvre le journal, Roquentin constate qu'« il y avait quelque chose [qu'il] a $v u$ et qui

\footnotetext{
${ }^{1}$ Michel Contat et Michel Rybalka soulignent que La nausée de Sartre a influencé le destin même du mot "nausée ", qui désormais ne peut plus ne pas évoquer le célèbre roman de 1938 : « [...] le mot de nausée a en quelque sorte changé de sens grâce au roman de Sartre : il suffit aujourd'hui de lui mettre une majuscule pour qu'il évoque non plus le malaise physique ou les vomissements, mais l'angoisse existentielle» (Contat-Rybalka 1995, 1668).
} 
[1]'a dégoûté » (Sartre 1938, 6, je souligne), alors que, au bord de la mer, il tenait dans la main un galet, " plat, sec sur tout un côté, humide et boueux sur l'autre » (ibid.). Après la description d'une série d'objets auxquels sont attribuées des perturbantes propriétés animales (cf. ibid., pp. 8-15)², par le biais d'une opération réflexive, Roquentin comprends - voit - l'origine de son trouble :

Les objets, cela ne devrait pas toucher, puisque cela ne vit pas. On s'en sert, on les remet en place, on vit au milieu d'eux : ils sont utiles, rien de plus. Et moi, ils me touchent, c'est insupportable. J'ai peur d'entrer en contact avec eux tout comme s'ils étaient des bêtes vivantes.

Maintenant je vois ; je me rappelle mieux ce que j'ai senti, l'autre jour, au bord de la mer, quand je tenais ce galet. C'était une espèce d'écœurement douceâtre. Que c'était donc désagréable ! Et cela venait du galet, j'en suis sûr, cela passait du galet dans mes mains. Oui, c'est cela, c'est bien cela : une sorte de nausée dans les mains. (Sartre 1938,16)

Le malaise qui affecte Roquentin est strictement lié à une radicale mutation concernant les objets du monde, qui ne sont plus 'simplement' " utiles » et disponibles, mais qui semblent presque assumer des pouvoirs et des propriétés subjectives. Ce passage ne peut ne pas évoquer les concepts heideggériens d'«outil » et d'« ustensilité » («Zeug » et «Zuhandenheit», cf. Heidegger 1927, $\S \S 15-18$ et 69$)^{3}$, spécialement dans la corrélation que leur « effondrement » entretient avec l'« affection fondamentale » («Befindlichkeit ») qu'est le vécu de l'angoisse (cf. Heidegger 1927, § 40). En ce sens, de même qu'il en est pour l'angoisse chez Heidegger, la nausée serait en mesure de révéler «le monde comme tel »

\footnotetext{
${ }^{2}$ Le loquet d'une porte est doué d'« une sorte de personnalité »; la main du personnage que Roquentin rencontre toujours à la bibliothèque communale - dénommé " l'Autodidacte » - ressemble à " un gros vers blanc »; un bocal de bière arrive jusqu'à faire « peur »...

${ }^{3}$ L'attention de Sartre au thème heideggérien de l'« ustensilité » est relevée par Grégory Cormann, dans le cadre d'un dense et important article sur lequel je reviendrai et dans lequel l'auteur se consacre à " reconstituer la cohérence que Sartre donne à un ensemble d'interventions des thèses heideggériennes dans le champs intellectuel français », sans tenter de prouver l'inadéquation de Sartre à l'Heidegger « réel ». Cf. Cormann 2014, 142144, en particulier la note $\mathrm{n} .9$, au sein de laquelle Cormann renvoie au monumental travail d'Alain Flajoliet La première philosophie de Sartre, où ce dernier analyse en profondeur le rapport et la distance que le premier Sartre entretient avec la pensée de Heidegger, tout en relevant l'importance, pour l'évolution de la pensée sartrienne, de la réception de Was ist Metaphysik et des passages de Sein und Zeit sur l'«ustensilité» et sa perturbation, ainsi que sur l'angoisse (Flajoliet 2008, 268-269, 776).
} 
(ibid.), l'être dans sa « totalité » (Heidegger 1931, 19), ce qui est en effet déclaré par Roquentin :

Je n'étais pas surpris, je savais bien que c'était le Monde, le Monde tout nu qui se montrait tout d'un coup, et j'étouffais de colère contre ce gros être absurde. On ne pouvait même pas se demander d'où ça sortait, tout ça, ni comment il se faisait qu'il existât un monde, plutôt que rien. Ça n'avait pas de sens, le monde était partout présent, devant, derrière. Il n'y avait rien eu avant lui. Rien. Il n'y avait pas eu de moment où il aurait pu ne pas exister. C'est bien cela qui m'irritait : bien sûr il n'y avait $a u$ cune raison pour qu'elle existât, cette larve coulante. Mais il n'était pas possible qu'elle n'existât pas. C'était impensable : pour imaginer le néant, il fallait qu'on se trouve déjà là, en plein monde et les yeux grand ouverts et vivants ; le néant ça n'était qu'une idée dans ma tête [...]. Je criais « quelle saleté, quelle saleté ! » [...], mais elle tenait bon et il y en avait tant, des tonnes et des tonnes d'existence, indéfiniment : j'étouffais au fond de cet immense ennui. (Sartre 1938, 159)

Toutefois, la correspondance entre angoisse heideggérienne et nausée sartrienne ne va pas si simplement de soi, spécialement dans la mesure où la manifestation du néant ne caractérise point cette dernière, alors qu'elle se configure comme un aspect typique de la première, comme il émerge, en particulier, des analyses de l'angoisse que Heidegger présente en 1929 dans Was ist metaphysik? (et ceci, d'ailleurs, semble bien être confirmé par le passage cité, où Sartre liquide la question même sur laquelle se conclut la conférence heideggérienne) ${ }^{4}$. En effet, comme il a été relevé (cf. Cormann 2014, 165 et suiv.), l'opération sartrienne à l'égard de la question de l'affectivité est complexe; elle s'inscrit dans la lignée de la tortueuse réception - et théorique et textuelle - française de la phénoménologie et semble bien prendre le relais du projet lévinassien d'une " herméneutique de la facticité » (Lévinas 1932, 417), présenté au cours de la première moitié des années $1930^{5}$, à

4 « Pourquoi l'Être plutôt que le néant? », dans la traduction par Henri Corbin. Cf. Heidegger 1931, 27 et supra, note 5.

${ }^{5}$ L'expression est utilisée par Lévinas en 1932, dans un des premiers articles d'introduction de la philosophie heideggérienne en France, Martin Heidegger et l'ontologie. Comme le précise Cormann, Lévinas désigne par cette formule « la manière dont le Dasein se saisit ou manque les possibilités qui se présentent à lui, la manière dont il ressaisit dans un projet de soi les possibilités au milieu desquelles il se trouve déjà jeté » (Cormann 2014, 171). Toutefois, le texte lévinassien qui tente proprement d'entreprendre ce projet - comme l'a remarqué Jacques Rolland dans sa célèbre introduction - est De l'évasion (Lévinas, 1935-1936), où "Lévinas s'attarde sur la Geworfenheit », pour " décrire une situation où l'existence ne trouve plus en elle une propension allant au-delà de la situation imposée, une situation dans laquelle l'être-jeté paralyse en 
la suite de la première - et décisive - traduction de Heidegger en français: à savoir, Qu'est-ce que la métaphysique ?, par Henri Corbin $^{6}$. Avec ses premiers travaux philosophiques (et phénoménologiques), Sartre aurait donc élaboré à sa manière une réponse à la problématique qui principalement se répand dans le milieu intellectuel français recevant la pensée heideggérienne ; celle posée par la corrélation entre l'émotion et la situation (ou l'être situé), soit la question concernant l'affectivité et l'effectivité, la Befindlichkeit et la Faktizität ${ }^{7}$.

Dans le cadre de cette lecture, trois œuvres du premier Sartre s'avèrent pertinentes : La transcendance de l'Ego (1937), La nausée (1938) et l'Esquisse pour une théorie des émotions (1939), car dans leur complexe elles abordent (entre autre) une série d'émotions, en relation à l'être situé et jeté qui concerne et caractérise l'existence humaine dans le monde. L'Esquisse pour une théorie des émotions est entièrement dédiée à la question de l'affectivité et considère les émotions comme des conduites d'évasion par rapport à une situation devenue trop " difficile », par le biais de la «transformation du monde » (Sartre 1939a, 43). Ce texte a pour but d'analyser l'« essence "a priori" d'être humain » (ibid., 12 et suiv.) ${ }^{8}$, car «l'émotion signifie à sa manière le tout de la

quelque sorte toute possibilité de se projeter » (Rolland 2011, 26-28 ; Cormann 2014, 165). Comme l'a souligné Cormann, ce texte - ainsi que toute l'atmosphère intellectuelle qui l'entoure et l'accompagne - devait être connu par Sartre, car il est publié dans la livraison de 1936 des "Recherches Philosophiques", soit la revue qui a certainement conté le plus dans la réception française de la phénoménologie et qui, en outre, accueille le tout premier travail philosophique de Sartre, La transcendance de l'Ego, dans le numéro qui suit celui où se trouve De l'évasion (cf. Cormann 2014, 145-146). En ce sens, la question - qui excède largement le propos de cet article - d'une possible influence du texte de Lévinas De l'évasion sur le roman de Sartre, au sujet du vécu de la nausée, doit être insérée dans un cadre plus ample et ne pas être considérée comme directe.

${ }^{6}$ L'article est paru en 1931 dans le numéro 8 de la revue " Bifur », dans lequel paraissait aussi un des premiers textes philosophiques de Sartre : La légende de la vérité (Sartre 1931).

${ }^{7}$ En s'apprêtant à analyser cette question, Cormann reprend le bel article où Jean Greisch s'occupe de l'interprétation de la facticité heideggérienne de par Lévinas (spécialement dans le cadre de son article de 1932) et au sein duquel l'auteur souligne que " la principale originalité » lévinassienne « réside dans la corrélation étroite qu'elle établit entre l'affectivité et l'effectivité, la Befindlichkeit et la Faktizität», dont résulte une mise en relief de «la dimension dramatique ou tragique de la compréhension de l'existence » (cf. Greisch 2000, 189 et suiv. ; Cormann 2014, 169).

${ }^{8}$ La citation sartrienne semble bien renvoyer à l'Introduction et au $\S 9$ de Sein und Zeit, qui soulignent l'« urgence » d'une « tâche [...] à peine moindre que celle de la question de l'être elle-même: la libération de l'a priori qui doit nécessairement devenir visible pour 
conscience [...], de la réalité-humaine» ${ }^{9}$, de la " totalité humaine », " de l'homme en situation » (ibid., 16-18). La transcendance de l'Ego, par contre, analyse l'essence de la conscience humaine, en faisant abstraction (ou, plutôt, épochè) de son être-dans-le-monde, soit en dehors de tout rapport concret avec l'être, avec tout étant déterminé. Dans ce cadre, l'Ego lui-même est conçu comme un étant transcendant, un refuge inauthentique de la conscience dont la nature est d'être un " absolu non substantiel » (Sartre 1937, 103-107), absolument libre et spontané et qui, toutefois, nécessite de transcender l'être et d'y être en relation, pour exister. Et ce qui permet de révéler à la conscience, de façon réflexive, cette manière d'être en tant que néant d'être, c'est le "vertige » que suscite le vécu de l'angoisse (ibid., 118-122).

Chaque émotion ne joue donc pas le même rôle dans la phénoménologie de l'existence et dans l'ontologie sartrienne : certains vécus constituent une voie d'accès privilégiée à l'être ; d'autres, par contre, représentent une fuite face à ce dernier. À ce propos, le Grand OEuvre de 1943 est très clair lorsque, dès les toutes premières pages, il déclare que «l'Être nous sera dévoilé par quelque moyen d'accès immédiat, l'ennui, la nausée, etc., et l'ontologie sera la description du phénomène d'être tel qu'il se manifeste, c'est-à-dire sans intermédiaire » (Sartre 1943, 14). En ce sens, les vécus qui sont au cœur du roman de 1938 (la nausée bien évidemment - et l'ennui, considéré par Sartre comme synonyme de cette dernière $)^{10}$ sont effectivement en mesure de révéler le Monde dans sa totalité, l'Être ${ }^{11}$, de même qu'il en est pour

que la question “qui est l'homme?" puisse recevoir une élucidation philosophique. » (Heidegger 1927, § 9).

${ }^{9}$ Comme on le sait, « Réalité-humaine » est la première - très discutée et discutable traduction française de "Dasein », offerte par Corbin en 1931 dans Qu'est-ce que la métaphysique?.

10 Cf. supra, note 5. Sur l'équivalence posée entre nausée et ennui, il n'est certainement pas superflu de rappeler que le titre que Sartre aurait voulu donner à l'origine à son ouvrage - avant que l'éditeur, Gaston Gallimard, ne s'imposât - était " Mélancholia » (cf. Contat-Rybalka 1995, 1667-1668 ; Contat 2003). En ce sens, il est possible de considérer nausée et ennui - ou mélancolie - comme synonymes. Sur ce point, il n'est certainement pas inintéressant de souligner qu'une des très probables sources sartriennes sur le thème du dégoût (cf. infra, note 17), Aurel Kolnai, dans son texte de 1929 Der Ekel met explicitement en relation le dégoût et l'ennui, en se référant à la " saturation » que ressent, par exemple le personnage du roman de Gontcharov, Oblomov. Sur ce thème spécifique, d'ailleurs, Kolnai publie un texte en 1923 sur la célèbre Revue freudienne «Imago ». Cf. Kolnai 1923 ; 1929, 76-77 (l'édition citée de Der Ekel est celle italienne 2017).

11 Il est nécessaire de souligner le fait que, dans La nausée, le terme « être » est utilisé pour indiquer l'idéal auquel la conscience humaine aspire et qui, de fait, ne constituer 
l'angoisse heideggérienne. Toutefois, étant donné qu'au sein des travaux sartriens de cette même période figure l'analyse de l'angoisse et que sa fonction est celle de révéler, non pas l'être dans sa totalité, mais l'être de la conscience, la correspondance avec le procédé heideggérien ne doit pas être poussée trop loin (de même qu'il doit en être pour l'acception et la connotation du concept d'« Être »). Sur cette question, Sartre se prononce clairement dans ses Carnets de la drôle de guerre, rédigés dans cette même période :

Angoisse devant le Néant, avec Heidegger ? Angoisse devant la liberté, avec Kierkegaard? A mon sens, c'est une seule et même chose, car la liberté c'est l'apparition du Néant dans le monde. Avant la liberté, le monde est un plein qui est ce qu'il est, une grosse pâtée. Après la liberté, il y a des choses différenciées parce que la liberté a introduit la négation. [...] C'est par la liberté que nous pouvons imaginer, c'est-à-dire néantiser et thématiser à la fois des objets du monde. [...] L'angoisse devant le Néant n'est pas autre chose que l'angoisse devant la liberté ou, si l'on préfère, l'angoisse de la liberté devant elle-même. [...] Les angoisses devant le Néant du Monde, devant les origines de l'existant, sont dérivées et secondaires. Ce sont des problèmes qui apparaissent à l'éclairage de la liberté. Le monde en lui-même est et ne peut pas ne pas être. [...] Ainsi la saisie existentielle de notre facticité, c'est la Nausée, et l'appréhension existentielle de notre liberté, c'est l'Angoisse. (Sartre 1939b, 416-417)

L'angoisse dont il question spécialement dans La transcendance de l'Ego concerne l'appréhension de la liberté caractéristique de la

pas la réalité, c'est-à-dire l'« existence » - celle qui cause la « nausée ». Dans le Carnet Dupuis (cahier où Sartre, lors de sa permanence à Le Havre dans la première moitié des années 1930, prenait ses notes en vue de l'écriture de La nausée), on peut lire en effet: « distinction de l'existence et de l'être. Ce qui est n'existe pas, ce qui existe n'est pas. Exemple : une idée. Essence objective et essence formelle. » (Sartre 1995, 1684-1685). Dans L'être et le néant, par contre, ce qui correspond à l'existence décrite dans La nausée est nommé « être ». À ce sujet, Marteen Van Bureen retient - de façon peut être un peu trop schématique - que les significations des concepts d'« être » et d' « existence » utilisés dans La nausée et dans L'être et le néant constituent une structure chiasmatique (Van Bureen 2007). Cette lecture est un peu trop manichéenne, spécialement car et dans $L a$ nausée et dans le Grand CEuvre de 1943 figurent aussi des passages où l'« être » et «l'existence» correspondent et sont considérés comme synonymes: «j'avais la tête vide, ou tout juste un mot dans la tête, le mot "être". » (Sartre 1938, 150) ; « [...] l'En-soi originel [est] figé dans sa contingence et sa facticité et [... sa] caractéristique essentielle est qu'il est, qu'il existe [...]. » (Sartre 1943, p. 636). Il relève toutefois que l'ontologie présentée dans La nausée analyse l'« existence »- en considérant l' « être » comme un idéal - et que, dans L'être et le néant, l'« existence » qui a été décrite dans le roman de 1938 est identifiable comme " être » (plutôt dans la version décrite lors des passages sur le « visqueux » et sur l'« Antivaleur», dans le paragraphe sur « la qualité comme révélatrice de l'être », à la fin de l'ouvrage; cf. Sartre 1943, 661-667). 
conscience humaine, qui introduit le néant dans le monde ; la nausée, par contre, est suscitée par la saisie de la facticité humaine et, en ce sens, elle exprime parfaitement la corrélation mentionnée entre Befindlichkeit et Faktizität, tout en n'étant point en relation avec le concept de néant que révèle l'angoisse heideggérienne. En effet, ce qui cause la nausée - et différentie sa signification ontologique de celle l'angoisse - c'est la « plénitude » de l'existence, révélée dans sa nature la plus intime, c'est-à-dire dans toute son essentielle absurdité. Sur ce point Sartre ne manque jamais d'insister, en explicitant toujours le lien intrinsèque qui subsiste entre le concept de "plein » (Sartre 1938, 156-157 ; supra, note 14) qui caractérise l'existence et l'absurde. À ce sujet, un passage de La nausée est particulièrement explicite, spécialement dans la mesure où il est précédé par deux déclarations de Roquentin qui manifestent la conscience d'avoir appris la vérité concernant la question sur laquelle s'est ouvert le roman - la question de la vérité tout court:

Et tout d'un coup, d'un seul coup, le voile se déchire, j'ai compris, j'ai $v u$.

[...] Et puis j'ai eu cette illumination.

[...] Et puis voilà : tout d'un coup, c'était là, c'était clair comme le jour : l'existence s'était soudain dévoilée. Elle avait perdu son allure inoffensive de catégorie abstraite : c'était la pâte même des choses, cette racine était pétrie dans l'existence. Ou plutôt la racine, les grilles du jardin, le banc, le gazon rare de la pelouse, tout ça s'était évanoui ; la diversité des choses, leur individualité n'était qu'une apparence, un vernis. Ce vernis avait fondu, il restait des masses monstrueuses et molles, en désordre nues d'une effrayante et obscène nudité. (Sartre 1938, 150-151)

Les références au thème de la nudité, dévoilée et résultant d'une « illumination » capable d'offrir la vérité - opposée à l'« apparence » - sont autant d'évidents renvois à la méthode phénoménologique et à la conversion d'attitude impliquée par l'épochè (cf. supra, note 2). En ce sens, la perte d'« individualité » et de "diversité des choses », qui fond comme "un vernis » et s' " évanoui » est de toute évidence non seulement une reprise de la description que fait Heidegger de l'«évanescence totale» et de «l'évanouissement» de l'existant qui advient lors de l'affection fondamentale (Heidegger 1931, 19), mais une reformulation sartrienne du procédé husserlien de mise entre parenthèse du monde qui est présent «sous la main» («Vorhanden »; cf. Husserl 1913, § 32).

Ce que la nausée révèle en mettant hors-circuit l'individualité des étants est que, en-dessous de la surface du monde, de l'ap- 
parence, du « vernis », se trouve la vérité concernant l'exister, qui n'est au fond qu'une originaire indifférence. Et si, comme le veut Husserl, la phénoménologie est " une théorie descriptive de l'essence des vécus transcendantalement purs », qui parvient à des « connaissances absolues » par le biais de « ce qui dans les vécus réduits peut être saisi de façon eidétique dans une intuition pure » (ibid., § 75), alors la nausée sartrienne est expression de l'essence de l'existence (de même que les émotions décrites dans l'Esquisse analysent l'essence de l'être humain dans sa totalité, cf. supra, note 10). Cette essence s'avère paradoxalement inéssentielle, dans la mesure où toute possibilité d'individuation des étants disparait dans la dimension 'pâteuse' de l'existence (cf. supra, notes 14 et 15) ${ }^{12}$. Et cette inéssentialité n'est que la contingence qui, dès la fin des années 1920, a guidé le parcours philosophique de Sartre ${ }^{13}$. À ce sujet, Sartre est explicite et énonce, au cœur du roman, celle qui pourrait être définie comme une véritable - et paradoxale - loi de la contingence :

[...] je comprenais la Nausée, je la possédais. [...] L'essentiel c'est la contingence. Je veux dire que, par définition, l'existence n'est pas la nécessité. [...] Quand il arrive de s'en rendre compte, ça vous tourne le cœur et tout se met à flotter [...] : voilà la Nausée ; voilà ce que les Salauds [...] essaient de se cacher avec leur idée de droit. Mais quel pauvre mensonge : personne n'a de droit ; ils sont entièrement gratuits, comme les autres hommes, ils n'arrivent pas à ne pas se sentir de trop. Et en eux-mêmes,

${ }^{12}$ La caractérisation de l'exister originaire comme plénitude perturbante et dégoutante doit très probablement quelque chose aux recherches phénoménologique d'un élève de Husserl : Aurel Kolnai. En effet, dans son texte majeur - Der Ekel, publié en 1929 sur le Jahrbuch husserlien et, donc, très probablement lu par Sartre au cours de ses lectures phénoménologiques lors de son séjour à Berlin (cf. De Coorebyter 2000; Flajoliet 2008) - qui s'occupe d'offrir une inédite analyse phénoménologique du vécu du dégoût, Kolnai insiste sur certains éléments qui caractérisent de façon spécifique aussi la nausée sartrienne, spécialement dans sa différence par rapport à d'autres vécus, comme l'angoisse heideggérienne. Notamment, Kolnai insiste sur l'aspect de la plénitude et sur la dimension amorphe qui caractérisent l'objet dégoûtant : « on se trouve toujours devant une vie qui, malgré la note excessivement emphatique de la "plénitude", est appauvrie dans ses dimensions et abandonne sa structure d'ensemble pour déborder en suivant une seule ligne. Le fond intentionnel, les perspectives du regard, le caractère de totalité manquent dans cette situation et la vie est comprimée dans un "fluide" vital essentiellement uniforme [...]. » (Kolnai 1929, 91, je traduis). Sur la question de la possible influence de Kolnai sur Sartre, cf. Tedeschini 2016, 2017, 2018 ; sur le thème de la " proximité » - et donc de l'informe - dans les textes de Kolnai, cf. Fussi $2016 ; 2017$.

${ }^{13}$ Le récit de Simone de Beauvoir à ce sujet est connu : dans un entretien avec elle, Sartre raconte avoir eu l'intuition de la contingence à la sortie du cinéma, car le monde ne suivait pas la même nécessité qui, par contre, guidait avec évidence la pellicule (cf. de Beauvoir 1981, 199-200). 
secrètement, ils sont trop, c'est-à-dire amorphes et vagues et tristes. (Sartre 1938, 155)

La nausée est donc le vécu suscité par la manifestation de l'essence de l'existence en tant que contingence, manque absolu de nécessité, en un mot : absurdité. Cette corrélation entre nausée, existence et absurdité est, en outre, mise en évidence par l'usage des majuscules, toujours au sein d'un passage central du roman, où Roquentin déclare : « le mot d'Absurdité nait à présent sous ma plume [...et] sans rien formuler, je comprenais que j'avais trouvé la clé de l'Existence, la clé de mes Nausées, de ma propre vie. » (Sartre 1938, 152-153). La révélation de cette vérité advient à contre-courant de l'attitude ordinairement adoptée par ceux que Sartre nomme les "Salauds ", soit ceux qui persistent dans l'attitude naturelle, selon laquelle les choses du monde sont par essence - en « droit »-comme elles se montrent au quotidien.

C'est cette idée de « droit » que Sartre récuse ; l'idée selon laquelle le monde et la nature sont guidés par des lois et ont donc un sens précis, nécessaire et téléologiquement orienté. Pour Sartre, l'exister, dans son 'essence' authentique, excède - il est « de trop ${ }^{14}$ - tout principe rationnel, toute raison et ne peut être expliqué15: rien ne le précède - ni même le rien, le néant - et aucune finalité en est le guide. Une opposition se configure donc entre le monde des " salauds », celui de l'attitude naturelle, où règnent les lois et les présences certaines et rassurantes des étants « utilisables » et celui - authentique - que révèle la nausée : un monde qui trouble, à cause de l'effondrement de toutes les certi-

\footnotetext{
14 Dans le Carnet Dupuis, on peut lire : « la contingence n'est pas inhumaine, au contraire, trop humaine. Les mains de chair sur le front. L'inhumain = envers de la nécessité dirigée. » (Sartre 1995, 1685-1686). La contingence est donc inhumaine ; toutefois, l'humain - trop humain - ne consiste nullement en la nécessité, mais dans une recherche de celleci. Dans ces passages, le renvoi aux premières lignes de Menschliches, Allzumenschliches. Ein Buch für freie Geister semble assez évident. À ce sujet, certains interprètes ont souligné l'influence de Nietzsche sur le premier Sartre, ainsi que la possibilité de voir en Roquentin un Zarathustra 'à l'envers' (cf. de Coorebyter 2005, 252-261 ; Louette 1996, 3437).

15 Cette antipathie pour le rationalisme a conduit Sartre - dans ce contexte en particulier - à virer vers la déclinaison heideggérienne de la phénoménologie, plutôt qu'à rester fidèle à la stricte doctrine husserlienne, qui est d'ailleurs critiquée dans ce cadre. Un même tournant caractérise la toute première pensée lévinassienne (celle de la Théorie de l'intuition dans la phénoménologie de Husserl, premier livre en français sur le fondateur de la phénoménologie et premier texte lu par Sartre sur ce sujet), qui déclare vouloir abandonner la théorie en faveur de la vie (cf. Lévinas 1930,218) et qui a certainement influencé Sartre en ce sens.
} 
tudes dans la même matière indifférenciée et originaire. Sartre est encore explicite à ce sujet :

Dans un autre monde, les cercles, les airs de musique gardent leurs lignes pures et rigides. Mais l'existence est un fléchissement. [...L] e monde des explications et des raisons n'est pas celui de l'existence. Un cercle n'est pas absurde, il s'explique très bien par la rotation d'un segment de droite autour d'une de ses extrémités. Mais aussi un cercle n'existe pas. (Sartre 1938, 151-153)

La scission entre l'idéal et le réel, entre le droit, les principes et le fait est ici posée très clairement, de même que la différence - qui a guidé tout le procédé sartrien - entre la rigidité et la netteté de la forme et la mollesse de la matière qu'est l'exister originaire (et nauséabond). En cette séparation - traduisible dans les termes plus classiques de matière et de forme - consiste la contingence de l'existence originaire, par rapport à laquelle la prérogative de donation de sens («Sinngebung»; cf. Husserl 1913, § 55) de la conscience se trouve désarmée. Pour cette raison, l'aspiration de la conscience humaine - dont il a été dit qu'elle est caractérisée par la liberté - est celle de fonder le réel, en lui conférant un sens. Toutefois, dans L'être et le néant, Sartre explique clairement que cette tâche n'est qu'un idéal, apte à guider la création humaine, qui est orientée vers une perfection capable de compenser le dégoût, non sur le plan théorique et rationnel, mais sur celui esthétique.

[...] cette fusion doit être telle que l'abstrait soit fondement du concret et simultanément le concret fondement de l'abstrait; en d'autres termes, l'existence concrète « en chair et en os » doit être l'essence [...]. Ou, si l'on préfère, la forme doit être à elle-même - et totalement - sa propre matière. Et réciproquement la matière doit se produire comme forme absolue. Cette fusion impossible et perpétuellement indiquée de l'essence et de l'existence n'appartient ni au présent ni à l'avenir, elle indique plutôt la fusion du passé, du présent et de l'avenir, et se présente comme synthèse à opérer de la totalité temporelle. C'est la valeur, en tant que transcendance ; c'est elle que l'on nomme beauté. [...] À l'ordinaire, le beau, comme la valeur, [...] est implicitement appréhendé sur les choses comme une absence ; il se dévoile implicitement à travers l'imperfection du monde. (Sartre 1943, 235-236)

L'« imperfection du monde » dont parle Sartre est bien la laideur scandaleuse qui est la source de la nausée et qui se manifeste agressivement, en désarmant la conscience et la subjectivité, comme il est arrivé à Roquentin. La beauté, par contre, est origi- 
nairement absente de ce monde-ci et - en se révélant avant tout « implicitement »- se configure comme un ouvrage à opérer. Et La nausée - véritable ouvrage œuvré - est déjà une réponse à cette mission, selon les principes qui seront, plus tard, explicités dans le manifeste sartrien de l'engagement littéraire Qu'est-ce que la littérature? (Sartre 1947). En effet, à ce propos, dans ses Carnets de la drôle de guerre, Sartre écrit : " la différence essentielle entre Antoine Roquentin et moi, c'est que moi j'écris l'histoire d'Antoine Roquentin [...] » (Sartre 1939b, 594).

\section{Bibliographie}

Beauvoir de S., 1981 : La cérémonie des adieux suivi de Entretiens avec Jean-Paul Sartre, août-septembre 1974, Paris, Gallimard, 1987.

Contat M.-Rybalka M., 1995 : Notice à La nausée, dans Sartre J.-P., OEuvres romanesques, Paris, Gallimard, La Pléiade, 1995.

Contat M., 2003 : De Mélancholia à La nausée : la normalisation NRF de la contingence, dans « Genesis » 21, pp. 75-94.

Cormann G., 2014 : Sartre, Heidegger et les Recherches Philosophiques - Koyré, Lévinas, Wahl. Éléments pour une archéologie de la philosophie française contemporaine, dans Id.-Feron 0. (eds.), Questions anthropologiques et phénoménologiques. Autour du travail de Daniel Giovannangeli, Bruxelles, Ousia, 2014, pp. 141-174.

De Coorebyter V., 2000 : Sartre face à la phénoménologie : autour de "L'intentionnalité" et de "La transcendance de l'Ego", Bruxelles, Ousia.

De Coorebyter V., 2005 : Sartre avant la phénoménologie : autour de "La nausée" et de "La légende de la vérité", Bruxelles, Ousia. Flajoliet A., 2008 : La première philosophie de Sartre, Paris, Honoré Champion.

Fussi A., 2016 : Disgusto, paura, prossimità nell 'analisi fenomenologica di Aurel Kolnai, dans Centi B. (ed), Tra corpo e mente. Questioni di confine, Firenze, Le Lettere, 2016, pp. 257-280. 
Fussi A., 2017 : Kolnai and Aristotle on the Role of Proximity in Fear and Disgust, dans « Giornale di Metafisica » 2, 2017, pp. 1-17.

Greisch J., 2000 : Heidegger et Lévinas interprètes de la facticité, dans Marion J.-L. (ed), Lévinas E., Positivité et transcendance suivi de Lévinas et la phénoménologie, Paris, Puf, 2000, pp. 181-207.

Heidegger M., 1927 : Sein und Zeit, trad. it. Volpi F. sur la version de Chiodi P., Essere e Tempo, Milano, Longanesi, 2009 ; trad. fr. Vezin F., Etre et Temps, Paris, Gallimard, 1986.

Heidegger M., 1929 : Was ist Metaphysik?, trad. it. Volpi F., Che cos'è metafisica?, dans Segnavia, Milano, Adelphi, 1987, pp. 59-77 ; trad. fr. Corbin H., 1931: Qu'est-ce que la métaphysique?, dans « Bifur » 8, pp. 5-27.

Husserl E., 1913 : Ideen zu einer reinen Phänomenologie und phänomenologischen Philosophie, trad. it. Costa V., Idee per una fenomenologia pura e per una filosofia fenomenologica, Torino, Einaudi, 2002 ; trad. fr. Ricœur P., Idées directrices pour une phénoménologie, Paris, Gallimard, 1950.

Kolnai A., 1923 : Gontscharows "Oblomov», dans «Imago. Zeitschrift für Anwendung der Psychoanalyse auf die Geisteswissenschaften » 2, pp. 485-494.

Kolnai A., 1929 : Der Ekel, dans «Jahrbuch für Philosophie und Phänomenologische Forschung» 10, pp. 515-569 ; trad. it. Tedeschini M., Il disgusto, Milano, Marinotti, 2017.

Lévinas E., 1930 : Théorie de l'intuition dans la phénoménologie de Husserl, Paris, Vrin, 2010.

Lévinas E., 1932 : Martin Heidegger et l'ontologie, dans « Revue Philosophique de la France et de l'Étranger »1, pp. 395-431.

Lévinas E., 1935-1936 : De l'évasion, dans «Recherches Philosophiques » 5, pp. 373-392.

Louette J.-F., 1996 : Sartre contra Nietzsche, Grenoble, Pug, 1996. 
Rolland J., 2011 : Sortir de l'être par une nouvelle voie, dans Lévinas E., De l'évasion, Paris, Poche, 2011, pp. 13-88.

Sartre J.-P., 1931 : La légende de la vérité, dans « Bifur » 8, pp. 7796.

Sartre J.-P., 1936-1937 : La transcendance de l'Ego, dans « Recherches Philosophiques » 6, pp. 85-123.

Sartre J.-P., 1938 : La nausée, dans Contat M.-Rybalka M. (eds.), OEuvres romanesques, Paris, Gallimard, La Pléiade, 1995.

Sartre J.-P., 1939a : Esquisse d'une théorie des émotions, Paris, Hermann, 2010.

Sartre J.-P., 1939b : Carnets de la drôle de guerre, dans Louette J.F.Philippe G.-Simont J. (eds.), Les mots et autres écrits autobiographiques, Paris, Gallimard, La Pléiade, 2010.

Sartre J.-P., 1943 : L'Être et le néant. Essai d'ontologie phénoménologique, Paris, Gallimard, Tel, 1987.

Sartre J.-P., 1947 : Qu'est-ce que la littérature?, dans Situations II. Littérature et engagement, Paris, Gallimard, 1948.

Tedeschini M., 2016 : Distanti uno sputo. Disgusto e paura in Aurel Kolnai e Jean-Paul Sartre, dans «Itinera », 12, 2016, pp. 40-59.

Tedeschini M., 2017 : Una necessaria parzialità, dieci parole per Il disgusto, dans A. Kolnai, Il disgusto, Milano, Marinotti, 2017, pp. 525 .

Tedeschini M., 2018 : Il conflitto estetico. Teoria del disgusto, Roma, Lithos, 2018.

Van Bureen M., 2007 : Être et exister : le cas de La nausée, dans «Relief» 1, pp. 74-89. 